\title{
Generalizations of some irreducibility results by Schur
}

by

\author{
T. N. Shorey (Mumbai) and R. TiJdeman (Leiden)
}

1. Introduction. Let $a \geq 0$ and $a_{0}, a_{1}, \ldots, a_{n}$ be integers with

$$
\left|a_{0}\right|=\left|a_{n}\right|=1 \text {. }
$$

In this paper we study whether the polynomial

$$
f(x)=a_{n} \frac{x^{n}}{(n+a) !}+a_{n-1} \frac{x^{n-1}}{(n-1+a) !}+\cdots+a_{1} \frac{x}{(1+a) !}+a_{0} \frac{1}{a !}
$$

can have a factor of given degree over the rationals. In 1929 Schur [26], [27] proved that a polynomial of the form (1.2) satisfying (1.1) is irreducible if $a=0$ and also if $a=1$ unless $n+1$ is a power of 2 when it may have a linear factor or $n=8$ when it may even have a quadratic factor. Also for $a=2$ and many other values of $a$ the polynomial $f$ may have a linear factor. On the other hand, a factor of degree $>n / 2$ of $f$ has a cofactor of degree at most $n / 2$. Therefore we consider the question whether $f$ has a factor of degree $k$ with

$$
2 \leq k \leq n / 2,
$$

which we always assume unless specified otherwise. One of our results reads as follows.

THEOREM 1.1. Let $a$ and $k$ be integers such that

$$
2 \leq k \leq n / 2, \quad 0 \leq a \leq 3 k / 2 .
$$

Let $f(x)$ be given by (1.2) where $a_{0}, a_{1}, \ldots, a_{n}$ are integers satisfying (1.1). Assume that $f(x)$ has a factor of degree $k$. Then

$$
\begin{aligned}
(n, k, a) \in\{ & (6,2,3),(7,2,2),(7,2,3),(7,3,3),(8,2,1), \\
& (8,3,2),(12,3,4),(13,2,3),(22,2,3),(46,3,4),(78,2,3)\} .
\end{aligned}
$$

We shall show that all the cases listed in (1.3) can indeed be realized by some factorizations. The factorizations for the last two cases are due to 
the referee. See after the proofs of Lemma 4.1 and Theorem 1.1. In Theorem 4.4 we give a similar result for $k \geq 5$ with the upper bound 30 for $a$. In Theorem 5.1 we show that the requirement that $a$ is bounded by a constant times $k$ can be relaxed to

$$
a<c k \log k \frac{\log \log k}{\log \log \log k}
$$

for some constant $c$.

We also relax the restriction (1.1). Filaseta [9] showed in 1996 that if $a=0,\left|a_{0}\right|=1$ and $0<\left|a_{n}\right|<n$, then (1.2) is irreducible unless $\left(n, a_{n}\right) \in(6, \pm 5),(10, \pm 7)$. He also considered values of $a_{n}$ less than $n^{3 / 2} / \sqrt{2}$. He further proved a theorem due to Lam that (1.2) is irreducible if $a=0$ and $\operatorname{gcd}\left(a_{0} a_{n}, n !\right)=1$. Allen and Filaseta [1] computed for every $n$ the smallest integer $M=M(n)$ such that the polynomial $f(x)$ given by (1.2) with $a=1$, $\left|a_{0}\right|=1,\left|a_{n}\right|=M$ may be reducible for suitable coefficients $a_{i}$.

For an integer $\nu>1$, we denote by $\omega(\nu)$ and $P(\nu)$ the number of distinct prime divisors and the greatest prime factor of $\nu$, respectively. Further we put $\omega(1)=0$ and $P(1)=1$. We obtain the following result for the situation that $a_{0}= \pm 1$ and $a_{n}$ is a prime power, or conversely.

TheOREM 1.2. Let $f(x)$ be given by (1.2). Let $n \geq 1,2 \leq k \leq n / 2$, $0 \leq a \leq .75 k$ and $\omega\left(a_{n} a_{0}\right)=1$. Assume that $f(x)$ has a factor of degree $k$. Then $k \leq 5$ unless $(n, k, a) \in\{(22,9,6),(23,9,5),(24,9,4)\}$ and $P\left(a_{0} a_{n}\right)$ $=23$. If $3 \leq k \leq 5$, then $n+a \leq 32$ unless

$$
(n, k, a) \in\{(47,4,3),(48,4,3),(48,3,2),(79,3,2),(80,3,2),(125,4,3)\} .
$$

If $k=2$, then $a=1$.

Theorem 7.1 provides a result in case $a_{n}$ has more than one prime factor.

For real $x$ and integer $j$ we write $(x)_{j}=x(x+1) \cdots(x+j-1)$. We can express $f(x)$ from (1.2) by

$$
a ! f(x)=\sum_{j=0}^{n} a_{j} \frac{x^{j}}{(a+1)_{j}} .
$$

Since every product of $j$ consecutive integers is divisible by $j$ !, the hypergeometric polynomial

$$
g_{a, b, c}(x):=\sum_{j=0}^{n} \frac{(a)_{j}}{(b)_{j}(c)_{j}} x^{j} \quad(a, b, c \in \mathbb{Z})
$$

with $c=1, b=a+1$ is a special case of $f(x)$ in (1.4) by choosing $a_{j}=$ $(a)_{j} /(c)_{j}$. Some orthogonal polynomials can be expressed in the form (1.5) 
such as the Laguerre polynomials

$$
L_{n}^{(\alpha)}(x):=\sum_{j=0}^{n} \frac{(n+\alpha) \cdots(j+1+\alpha)}{j !(n-j) !}(-x)^{j}=\frac{(\alpha+1)_{n}}{(1)_{n}} g_{-n, \alpha+1,1}(x),
$$

where $n$ is a positive integer and $\alpha$ a complex number. Schur [26] proved in 1929 that $L_{n}^{(0)}(x)$ is irreducible for all $n$, and in 1931 that $L_{n}^{(1)}(x)$ is irreducible for all $n$ too [28]. Filaseta and Lam [12] proved the irreduciblity for all but finitely many $n$ of

$$
\mathcal{L}_{n}^{(\alpha)}(x):=\sum_{j=0}^{n} a_{j} \frac{(n+\alpha) \cdots(j+1+\alpha)}{j !(n-j) !}(-x)^{j}
$$

where $\alpha$ is a fixed rational number which is not a negative integer and $a_{0}, a_{1}, \ldots, a_{n}$ are any integers with $\left|a_{0}\right|=\left|a_{n}\right|=1$. Filaseta, Kidd and Trifonov [11] proved that either $L_{n}^{(n)}(x)$ is irreducible or it is a linear polynomial times an irreducible polynomial of degree $n-1$ and the latter possibility is excluded when $n \equiv 2(\bmod 4)$ with $n>2$. This settled completely the inverse Galois problem that for every positive integer $n$, there exists an explicitly given Laguerre polynomial of degree $n$ whose Galois group is the alternating group $A_{n}$.

In 2008 Filaseta, Finch and Leidy [10] proved that $L_{n}^{(\alpha)}(x)$ is irreducible for $0 \leq \alpha \leq 10$ unless $(n, \alpha) \in\{(2,2),(4,5),(2,7)\}$ in which cases $L_{n}^{(\alpha)}(x)$ is divisible by $x-6$. A consequence of Theorem 4.4 of the present paper is that for $5 \leq k \leq n / 2,0 \leq \alpha \leq 30$ the polynomial $L_{n}^{(\alpha)}(x)$ has no factor of degree $k$. We refer to [10] for a survey of irreducibility results connected with orthogonal polynomials and, in particular, Laguerre polynomials.

First we restrict our attention to integral values of $\alpha$. We extend the range of $a$ and $c$ as follows.

Theorem 1.3. Let $\epsilon>0$ and $1 \leq k \leq n / 2$. Assume

$$
a=-n-s \text { with } s \geq 0, b=\alpha+1, c \geq 1, s+c<\left(\frac{1}{3}-\epsilon\right) k
$$

where $c, n, s$ and $\alpha$ are integers with $0 \leq \alpha \leq k$. Suppose that $g_{a, b, c}(x)$ is divisible by a factor of degree $k$. Then $k$ is bounded above by an effectively computable number depending only on $\epsilon$.

Consider the Bessel polynomials

$$
y_{n}(x)=\sum_{j=0}^{n} \frac{(n+j) !}{2^{j}(n-j) ! j !} x^{j} .
$$


A simple calculation gives

$$
(-x)^{n} y_{n}\left(\frac{2}{x}\right)=n ! L_{n}^{(-2 n-1)}(x)=(-2 n)_{n} g_{-n,-2 n, 1}(x) .
$$

In 2002 Filaseta and Trifonov [13] proved that $L_{n}^{(-2 n-1)}(x)$, hence $y_{n}(x)$, is irreducible for all $n$. We also consider factors of functions $g_{-n, b, 1}$ for negative $b$. The irreducibility of $g_{-n,-n-s, 1}$ has been proved by Schur [26] for $s=0$, by Hajir [15] for $s=1$, by Sell [29] for $s=2$, and for $3 \leq s \leq 7$ and $n \geq n_{0}(s)$ by Hajir [16]. We consider $s$ bounded in terms of $k$ and settle the following result.

TheOREM 1.4. Let $2 \leq k \leq n / 2$. Let $g_{a, b, c}(x)$ be given by (1.5) such that $a=-n, b=\alpha+1, c=1$ where $\alpha=-n-s-1$ and $s$ is an integer with $0 \leq s \leq .95 k$. Then $g_{a, b, c}(x)$ has no factor of degree $k$.

So far we have been assuming that $a$ is an integer. In Section 10 we study the case of rational $a$. Let $a>0$ be a rational number such that

$$
a=u+\frac{\alpha}{\beta}
$$

where $u, \alpha, \beta$ are integers with $u \geq 0,0<\alpha<\beta$ and $\operatorname{gcd}(\alpha, \beta)=1$. Put

$$
(\alpha)_{\beta, m}=\alpha(\alpha+\beta) \cdots(\alpha+(m-1) \beta) .
$$

Hence $(\alpha)_{1, m}=(\alpha)_{m}$. Further put

$F(x):=F_{a}(x):=a_{n} \frac{\beta^{n} x^{n}}{(\alpha)_{\beta, n+u}}+a_{n-1} \frac{\beta^{n-1} x^{n-1}}{(\alpha)_{\beta, n-1+u}}+\cdots+a_{1} \frac{\beta x}{(\alpha)_{\beta, 1+u}}+a_{0} \frac{1}{(\alpha)_{\beta, u}}$ where $a_{0}, a_{1}, \ldots, a_{n} \in \mathbb{Z}$ with $\left|a_{0}\right|=\left|a_{n}\right|=1$.

The height $H(a)$ of a non-zero rational number $a$, in its reduced form, is defined as the maximum of the absolute values of its numerator and denominator. Then an analogue of Theorem 1.1 for the rational case is as follows.

THEOREM 1.5. Let $2 \leq k \leq n / 2$ and assume that $F(x)$ has a factor of degree $k$. Then there exist effectively computable absolute constants $k_{0}$ and $C_{1}>0$ such that for $k \geq k_{0}$, we have

$$
H(a) \geq C_{1} \log \log k .
$$

For further results in this direction we refer to Schur [27, and Allen and Filaseta [2]. In a recent paper Finch and Saradha [14] have given more precise information in case $\beta=2$.

In our proofs we follow the $p$-adic method of Coleman and Filaseta. See Lemma 2.13 and Corollary 2.14. This has been combined with a method arising out of a theorem of Sylvester that a product of $k$ consecutive positive integers each exceeding $k$ is divisible by a prime greater than $k$. See Lemmas 2.2-2.5, 2.7-2.9, 2.13 and Theorem 2.12. Further we need some results from 
prime number theory and factors of composite numbers; see Lemmas 2.6, 2.10 and 2.11. For the results from prime number theory we refer to [4] and [17. We also use the theory of linear forms in logarithms in the proofs of Theorems 5.1 and 10.2. In many cases we show that the exceptions in our theorems are unavoidable by giving examples of factorizations.

2. Lemmas. Let $x, k$ be positive integers with $x \geq 2 k$. We put ${ }_{k}(x)=x(x-1) \cdots(x-k+1) \quad$ and $\quad(x)_{k}=x(x+1) \cdots(x+k-1)$. Hence $(x)_{k}={ }_{k}(x+k-1)$.

LEMMA 2.1.

(a) If $k>4, x>42$ and $P\left({ }_{k}(x)\right) \leq 41$, then $(x, k)=(290,6)$ or $k=5$ and $x \in\{52,58,66,78,156,289,290,496,1521\}$.

(b) For $x>100$ the inequality $P\left({ }_{3}(x)\right) \leq 29$ is possible only if $x \in\{116,117,121,145,154,162,170,171,176,209,210,232,290,324,325$, $352,392,442,495,552,784,1276,2002,2025,2432,3250,9802,13312\}$.

(c) For $x>100$ the inequality $P\left({ }_{3}(x)\right) \leq 19$ is possible only if $x \in\{121,154,170,171,210,325,352,442,2432\}$.

(d) For $x>16$ the inequality $P\left({ }_{3}(x)\right) \leq 13$ is possible only if $x \in\{22,26,27,28,50,56,65,66,100,352\}$.

(e) For $x>16$ the inequality $P\left({ }_{4}(x)\right) \leq 13$ is possible only if

$$
x \in\{27,28,66\} .
$$

(f) For $x>100$ the inequality $P(x(x-1)) \leq 7$ is possible only if

$$
x \in\{126,225,2401,4375\} .
$$

(g) For $x>100$ the inequality $P(x(x-2)) \leq 7$ is possible only if

$$
x \in\{128,162,245\} .
$$

Proof. (a) We use Table IA of [22]. We collect all the numbers for which $x, x-1, x-2, x-3, x-4$ occur in the union of the tables for $t \leq 13$. (b) Check whether $x$ and $x-1$ are both in the tables for $t \leq 10$. (c) Use (b). (d) Use (c). (e) Use (d). (f) Use Table IA of [22] for $t \leq 4$. (g) Use Table IIA of 22$]$ for $t \leq 4$.

Lemma 2.2. Let $x \geq 2 k \geq 4$. Then

$$
P\left({ }_{k}(x)\right)>1.8 k
$$

unless $(x, k) \in\{(9,2),(10,3),(9,4),(10,4),(27,13),(28,13)\}$ or $x=2 k$ with $k \in\{2,3,4,5,8,11,13,14,18,63\}$. 
Proof. For $k=2$, the assertion follows from Lemma 2.1(f). For $k \geq 3$ see [20, Corollary 1] $=[18$, Corollary 1.3.2].

Lemma 2.3. If $x \geq 2 k \geq 4$, then

$$
P(k(x))>1.95 k
$$

unless $(x, k) \in\{(4,2),(9,2),(10,3)\}$ or $x \in\{2 k, \ldots, 2 k+h-1\}$ for $k \in E_{h}$ with $1 \leq h \leq 11$ where

$$
\begin{aligned}
E_{11}:=E_{10}:= & \{58\}, \quad E_{9}:=E_{8}:=\{58,59\}, \quad E_{7}:=E_{6}:=\{58,59,60\}, \\
E_{5}:=E_{4}:= & E_{6} \cup\{12,16,46,61,72,93,103,109,151,163\}, \\
E_{3}:=E_{2}:= & E_{4} \cup\{4,7,10,13,17,19,25,28,32,38,43,47,62, \\
& 73,94,104,110,124,152,164,269\}, \\
E_{1}:=E_{2} \cup\{3,5,6,8,9,11,14,15,18,20,23,26,29,33,35,39, & \\
& 41,44,48,50,53,56,63,68,74,78,81,86, \\
& 89,95,105,111,125,146,153,165,173,270\} .
\end{aligned}
$$

Proof. See [20, Theorem 2] $=[18$, Theorem 1.3.1].

Lemma 2.4. If $k \geq 2$ and $x \geq \max \left(2 k+13, \frac{541}{262} k\right)$, then

$$
P(k(x))>2 k \text {. }
$$

Proof. See [20, Theorem 1(a)] = [18, Theorem 1.3.3(a)].

LEMMA 2.5 .

(a) Between 69 and 7000 there is no block of 21 consecutive integers each of which is composed of primes $\leq 85$.

(b) Let $21 \leq k \leq 25$ and $x>k$. Assume that $P\left({ }_{k}(x)\right) \leq 2.5 k$. Then $x \leq 90$.

Proof. (a) By direct calculation.

(b) By deleting the terms divisible by $11 \leq p \leq 2.5 k$, we check that there are at least five terms in ${ }_{k}(x)$ composed of $2,3,5$ and 7 . For each prime we omit a term which contains the highest power of that prime. There remains a term $x^{*}$ in ${ }_{k}(x)$ divisible only by primes $2,3,5$ and 7 such that

$$
x \leq x^{*}+24 \leq 16 \cdot 9 \cdot 5 \cdot 7+24=5064 .
$$

Note that $2.5 k \leq 85$. Now apply part (a).

Lemma 2.6. Let $p_{1}=2<p_{2}<\cdots$ denote the sequence of primes. Then

$$
p_{i+1}-p_{i} \leq \begin{cases}6 & \text { if } p_{i}<89, \\ 14 & \text { if } p_{i}<523, \\ 34 & \text { if } p_{i}<9551, \\ 52 & \text { if } p_{i}<31397\end{cases}
$$


and

$$
p_{i+2}-p_{i} \leq 24 \quad \text { if } p_{i}<541 .
$$

Proof. By direct calculation (cf. Lander and Parkin [21]).

Lemma 2.7. If $20 \leq k<x \leq 12 k$ and $x \geq 60$, then

$$
P\left({ }_{k}(x)\right)>x-5 k / 6 \text {. }
$$

Proof. We put $x=1.072 X$. Then we observe that $x-5 k / 6<X$ since $x \leq 12 k$. By Lemma 2.6, for $60 \leq x \leq 128$ the gaps between consecutive primes are at most 14 , which is $\leq 5 k / 6$. Further $X \geq 120$ when $x>128$. It is a direct consequence of [1, Lemma 3] that for $X \geq 120$ there is a prime in $[X, 1.072 X]$. Thus $(x-5 k / 6, x]$ also contains a prime for $x>128$.

We write $\omega(x)$ for the number of distinct primes which divide $x$.

LEMMA 2.8.

(a) For $k \geq 3$ and $x \geq 2 k$, we have

$$
\omega\left({ }_{k}(x)\right) \geq \begin{cases}\min \left(\pi(k)+\left[\frac{3}{4} \pi(k)\right]-1+\delta(k), \pi(2 k)-1\right) & \text { for } k \leq 293, \\ \pi(k)+\left[\frac{3}{4} \pi(k)\right]-1 & \text { for } k>293,\end{cases}
$$

where $\delta(k)=0$ for $k \geq 17, \delta(k)=1$ for $7 \leq k \leq 16$ and $\delta(k)=2$ for $3 \leq k \leq 6$.

(b) If $x>\frac{29}{12} k-1$ and $(x, k) \neq(9,4)$, then

$$
\omega\left({ }_{k}(x)\right) \geq \pi(2 k) .
$$

Proof. (a) [19, Corollary 1] $=$ [18, Theorem 1.2.1 and Corollary 1.2.2].

(b) $[19$, Theorem 2] $=[18$, Theorem 1.2.4].

Lemma 2.9. Let $x \geq k^{2}$ and $k \geq 19$. Assume $f_{1}<\cdots<f_{\mu}$ are all integers in $[0, k)$ satisfying

$$
P\left(\left(x-f_{1}\right) \cdots\left(x-f_{\mu}\right)\right) \leq k .
$$

Then $\mu<k-\pi(2 k)+\pi(k)$.

Proof. [20, Lemma 4] $=$ [18, Lemma 6.1.6].

LEMMA 2.10.

(a) For any integer $x \geq 599$ we have

$$
\frac{x}{\log x}\left(1+\frac{0.992}{\log x}\right) \leq \pi(x) .
$$

(b) For any integer $x>1$ we have

$$
\pi(x) \leq \frac{x}{\log x}\left(1+\frac{1.2762}{\log x}\right) .
$$


(c) For any integer $x>1$ we have

$$
p_{x}>x \log x
$$

where $p_{x}$ denotes the $x$ th prime.

Proof. For (a) and (b) see [6] or [7], for (c) see [24, p. 69].

Lemma 2.11. For any integer $x>1$ we have

$$
\sqrt{2 \pi x} e^{-x} x^{x} e^{1 /(12 x+1)}<x !<\sqrt{2 \pi x} e^{-x} x^{x} e^{1 /(12 x)} .
$$

Proof. See [23].

For $s$ a non-negative integer we put ${ }_{k}(x)^{(s)}$ to be some product obtained from ${ }_{k}(x)$ by omitting any $s$ terms.

TheOrem 2.12. Let $k \geq 10$ and $x \geq 2 k$. Then

$$
P\left({ }_{k}(x)^{(1)}\right)>1.5 k \quad \text { or } \quad(x, k) \in\{(26,13),(27,13),(28,13),(28,14)\} .
$$

Proof. We find by direct calculation that the only solutions with $x<62$ are given by the exceptions in (2.1). Let $10 \leq k<19$ and $x \geq 62$. Suppose (2.1) does not hold. Then $P\left({ }_{k}(x)^{(1)}\right) \leq 27$. Hence at least five consecutive integers are composed of primes at most 27 . This is excluded by Lemma $2.1(\mathrm{a})$.

Thus $k \geq 19$ and $x \geq 62$. Then, by Lemmas 2.9 and $2.8(\mathrm{a})$, the number of the integers $i$ such that $x-i$ is a term in $k_{k}(x)^{(1)}$ satisfying $P(x-i)>k$ is at least $\pi(2 k)-\pi(k)$ if $x \geq k^{2}, \min ([3 \pi(k) / 4]-2, \pi(2 k)-\pi(k)-2)$ if $x<k^{2}, k \leq 293$ and $[3 \pi(k) / 4]-2$ if $x<k^{2}, k>293$. Further we observe, by Lemma 2.10 (a),(b) for $k>2000$ and direct computation for smaller $x$, that

$$
\pi(2 k)-\pi(k) \geq[3 \pi(k) / 4]-2 \quad \text { for } k>284 .
$$

Therefore, by Lemma 2.10(c),

$$
P\left({ }_{k}(x)^{(1)}\right) \geq p_{K}>K \log K
$$

where

$$
K:=K(k)= \begin{cases}\min ([3 \pi(k) / 4]+\pi(k)-2, \pi(2 k)-2) & \text { if } k \leq 293 \\ {[3 \pi(k) / 4]+\pi(k)-2} & \text { if } k>293 .\end{cases}
$$

We check by using exact values of $\pi$ function that $p_{K}>1.5 k$ for $k \leq 5393$ unless $k \in\{20,21,28,29\}$. For $k>5393$, we derive from Lemma 2.10(a) that $K \log K>1.5 k$. Hence

$$
P\left({ }_{k}(x)^{(1)}\right)>1.5 k \text {. }
$$

Let $k \in\{20,21,28,29\}$. We apply Lemma 2.9 when $x>k^{2}$ and Lemma 2.8(b) when $x<k^{2}$. Then the assertion follows if $x>\frac{29}{12} k-1$ since

$$
P\left(k(x)^{(1)}\right) \geq p_{\pi(2 k)-1}>1.5 k .
$$


Thus we assume $2 k \leq x \leq \frac{29}{12} k-1$. Then the assertion follows by direct computation.

For a prime $p$ and integers $a$ and $b$ with $a b \neq 0$ we make use of the $p$-adic notation

$$
\nu(a / b)=\nu_{p}(a / b)=e_{1}-e_{2} \quad \text { where } p^{e_{1}} \| a \text { and } p^{e_{2}} \| b .
$$

We define $\nu(0)=\infty$. Let $f(x)=\sum_{j=0}^{n} f_{j} x^{j} \in \mathbb{Z}[x]$ with $f_{0} f_{n} \neq 0$. Let

$$
S:=\left\{\left(0, \nu\left(f_{n}\right)\right),\left(1, \nu\left(f_{n-1}\right)\right), \ldots,\left(n-1, \nu\left(f_{1}\right)\right),\left(n, \nu\left(f_{0}\right)\right)\right\},
$$

a set of points in the extended plane. We consider the lower edges along the convex hull of the elements of $S$. The slopes of the edges are increasing when calculated from left to right. We define the Newton function with respect to the prime $p$ as the real function $F_{p}(x)$ on the interval $[0, n]$ which has the polygonal path formed by these edges as its graph. Hence $F_{p}(i)=\nu\left(f_{n-i}\right)$ for $i=0, n$ and at all points $i$ where the slopes of the edges change.

The next lemma plays a fundamental role in this paper. It is a refinement of a lemma due to Filaseta [8] which is based on a result of Dumas [5].

LEMMA 2.13. Let $k, n$ and $r$ be integers with $n \geq 2 k>0$. Let $h(x)=$ $\sum_{j=0}^{n} b_{j} x^{j} \in \mathbb{Z}[x]$ and let $p$ be a prime such that $p \nmid b_{n}$. Denote the Newton function of $h(x)$ with respect to $p$ by $H_{p}(x)$. Let $a_{0}, a_{1}, \ldots, a_{n}$ be integers with $p \nmid a_{0}, p \nmid a_{n}$. Put $f(x)=\sum_{j=0}^{n} a_{j} b_{j} x^{j}$. If $H_{p}(k)>r$ and $H_{p}(n)-H_{p}(n-k)<$ $r+1$, then $f(x)$ cannot have a factor of degree $k$.

Proof. Suppose $f(x)$ has a factor $g(x)$ of degree $k$. Let $G_{p}(x)$ be the Newton function with respect to $p$ of $g(x)$. Then $G_{p}(0)=0$ and $G_{p}(k)$ is an integer $m$, hence $G_{p}(x)$ has average slope $m / k$ on the interval $[0, k]$. It follows from Dumas's theorem ([5], cf. [8, Lemma 1]) that the Newton function $F_{p}(x)$ with respect to $p$ of $f(x)$ has segments corresponding to the irreducible factors of $g(x)$, which has average slope $m / k$. Since the slopes of the segments of $F_{p}(x)$ are increasing and $F_{p}(0)=0$, we obtain $F_{p}(k) \leq m$ and $F_{p}(n)-F_{p}(n-k) \geq m$. From the definition of $f$ we see that $F_{p}(x) \geq$ $H_{p}(x)$ for $0 \leq x \leq n, F_{p}(0)=H_{p}(0)=0$ and $F_{p}(n)=H_{p}(n)$. It follows that $F_{p}(k)>r$ and $F_{p}(n)-F_{p}(n-k)<r+1$. We conclude that $r<m$ and $m<r+1$. Since $m$ and $r$ are integers, this yields a contradiction.

Almost always we apply Lemma 2.13 in the following form which is equivalent with Lemma 2 of $[8]$.

Corollary 2.14. Let $k$ and $n$ be integers with $n \geq 2 k>0$. Let $h(x)=$ $\sum_{j=0}^{n} b_{j} x^{j} \in \mathbb{Z}[x]$ and let $p$ be a prime such that $p \nmid b_{n}$. Denote the Newton function of $h(x)$ with respect to $p$ by $H_{p}(x)$. Let $a_{0}, \ldots, a_{n}$ be integers with $p \nmid a_{0}, p \nmid a_{n}$. Put $f(x)=\sum_{j=0}^{n} a_{j} b_{j} x^{j}$. If $p \mid b_{j}$ for $j=0, \ldots, n-k$ and the left derivative of $H_{p}$ at $n$ is $<1 / k$, then $f(x)$ cannot have a factor of degree $k$. 
Proof. Since $p \mid b_{j}$ for $j=0, \ldots, n-k$, we have $H_{p}(k)>0$. Since the left derivative of $H_{p}$ at $n$ is $<1 / k$ and the derivative of $H_{p}$ can only change at integer points, we have $H_{p}(n)-H_{p}(n-k)<1$. Apply Lemma 2.13 with $r=0$.

LEMma 2.15. The positive integers $n$ for which

$$
P(12(n)) \leq 173
$$

are precisely those with either $n \leq 178$ or $n \in\{305,306,329,330\}$.

Proof. See Bauer and Bennett [3].

3. Some preliminary estimates. We introduce the following notation. Let $N=P_{1}^{a_{1}} P_{2}^{a_{2}} \cdots P_{r}^{a_{r}}$ where $P_{1}>P_{2}>\cdots>P_{r}$ are primes and $a_{1}, a_{2}, \ldots, a_{r}$ positive integers. Then we set

$$
P(N)=P_{1}(N)=P_{1}, \quad P_{2}(N)=P_{2}, \quad \ldots, \quad P_{r}(N)=P_{r} .
$$

Lemma 3.1. Let $l \geq 1, s \geq 0, \beta>1, \rho>1, k \geq 200, m=k \rho$ and $x \geq k^{\beta}$ be such that

$$
k-s-(\pi(m)-\pi(k))-(l-1)>\pi(k) .
$$

Assume that

$$
P_{l}\left(k(x)^{(s)}\right) \leq m
$$

Then

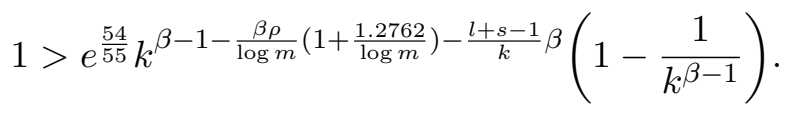

Proof. Assume (3.2). For every prime $p$ with $k<p \leq m$ dividing ${ }_{k}(x)^{(s)}$, at most one term of $k_{k}(x)^{(s)}$ is divisible by $p$ and we omit it. We also omit the terms of ${ }_{k}(x)^{(s)}$ divisible by $P_{j}^{\prime}=P_{j}\left(k(x)^{(s)}\right)$ for each $j<l$ with $P_{j}^{\prime}>m$. The remaining product is

$$
\left(x-f_{1}\right) \cdots\left(x-f_{\mu}\right) \quad \text { where } \quad 0 \leq f_{1}<\cdots<f_{\mu}<k
$$

and

$$
\mu \geq k-s-(\pi(m)-\pi(k))-(l-1)
$$

satisfying

$$
P\left(\left(x-f_{1}\right) \cdots\left(x-f_{\mu}\right)\right) \leq k .
$$

Notice that

$$
\left(x-f_{1}\right) \cdots\left(x-f_{\mu}\right) \mid k !\left(\begin{array}{l}
x \\
k
\end{array}\right) .
$$

Thus

$$
\left(x-f_{1}\right) \cdots\left(x-f_{\mu}\right) \leq k ! \prod_{p \leq k} p^{\left.\operatorname{ord}_{p}\left(\begin{array}{l}
x \\
k
\end{array}\right)\right)} \leq k ! \prod_{p \leq k} p^{\log x / \log p}=k ! x^{\pi(k)} .
$$


On the other hand

$$
\left(x-f_{1}\right) \cdots\left(x-f_{\mu}\right)>(x-k)^{\mu}=x^{\mu}\left(1-\frac{k}{x}\right)^{\mu} .
$$

By combining upper and lower estimates for $\left(x-f_{1}\right) \cdots\left(x-f_{\mu}\right)$ we derive

$$
k !>x^{\mu-\pi(k)}\left(1-\frac{k}{x}\right)^{\mu} .
$$

Since $x \geq k^{\beta}$ and $\pi(k)<\mu \leq k$ by (3.4) and (3.1), we obtain

$$
k !>k^{\beta(\mu-\pi(k))}\left(1-\frac{1}{k^{\beta-1}}\right)^{k} .
$$

By (3.4) and Lemma 2.10(b),

$$
\mu-\pi(k) \geq k-\pi(m)-l-s+1 \geq k-l-s+1-\frac{m}{\log m}\left(1+\frac{1.2762}{\log m}\right) .
$$

Thus, applying Lemma 2.11, we get

$$
\sqrt{2 \pi k} e^{-k} k^{k} e^{\frac{1}{12 k}}>k^{\beta\left(k-\frac{m}{\log m}\left(1+\frac{1.2762}{\log m}\right)-l-s+1\right)}\left(1-\frac{1}{k^{\beta-1}}\right)^{k} .
$$

By taking $k$ th roots on both the sides we have

$$
(2 \pi k)^{\frac{1}{2 k}} e^{-1} k e^{\frac{1}{12 k^{2}}}>k^{\beta\left(1-\frac{\rho}{\log m}\left(1+\frac{1.2762}{\log m}\right)-\frac{l+s-1}{k}\right)}\left(1-\frac{1}{k^{\beta-1}}\right) .
$$

Since $k \geq 200$, we see that

$$
\frac{\log (2 \pi k)}{2 k}+\frac{1}{12 k^{2}}<\frac{1}{55}
$$

Thus

$$
1>e^{\frac{54}{55}} k^{\beta-1-\frac{\beta \rho}{\log m}\left(1+\frac{1.2762}{\log m}\right)-\beta \frac{l+s-1}{k}}\left(1-\frac{1}{k^{\beta-1}}\right) .
$$

Corollary 3.2. Under the conditions of Lemma 3.1 with $\beta>1.1$ inequality (3.2) implies

$$
\beta\left(1-\frac{\rho}{\log m}\left(1+\frac{1.2762}{\log m}\right)-\frac{l+s-1}{k}\right)<1 .
$$

Proof. For $k \geq 200$ and $\beta>1.1$ we have $e^{54 / 55}\left(1-k^{-(\beta-1)}\right)>1$ and the assertion follows from (3.3).

Corollary 3.3. Let $l=1, s=0, \beta \geq 3 / 2, \rho=5 / 2, m=k \rho$ and $x \geq k^{\beta}$. Then (3.2) implies 


$$
k \leq \begin{cases}310 & \text { if } \beta=3 / 2, \\ 97 & \text { if } \beta=7 / 4, \\ 48 & \text { if } \beta=2, \\ 34 & \text { if } \beta=9 / 4 \\ 25 & \text { if } \beta=5 / 2 .\end{cases}
$$

Proof. Suppose the assumptions of Corollary 3.3 are satisfied. If $k>20$, then (3.1) and (3.6) are valid. Corollary 3.2 implies that $k \leq 3000$. By using (3.6) and the exact values of the function $\pi(x)$, we obtain the claimed bounds.

Corollary 3.4. Let $\epsilon>0, \beta=3 / 2, \rho=2$ and

$$
l+s<(1 / 3-\epsilon) k \text {. }
$$

Then (3.2) implies that $k$ is bounded by an effectively computable number depending only on $\epsilon$.

Lemma 3.5. Let $l=1, s=0, \rho=5 / 2, m=k \rho$ and $x \geq k^{3 / 2}$. Then (3.2) implies that $k \leq 20$.

Proof. Assume (3.2) holds with $k>20$. Then (3.7) is valid. Further we may suppose that none of the terms in ${ }_{k}(x)$ is prime, otherwise

$$
P(k(x))>x-k \geq k^{3 / 2}-k>5 k / 2
$$

contradicting (3.2). If $k>310$, then the assertion of the lemma follows from Corollary 3.3 .

Let $97<k \leq 310$. Then, by Corollary $3.3, x<k^{7 / 4} \leq 310^{7 / 4}<22903$ and we observe that $p_{i+1}-p_{i} \geq 85$, since none of the terms in ${ }_{k}(x)$ is prime. This is not possible since $p_{i+1}-p_{i} \leq 52$ for $p_{i}<31397$ by Lemma 2.6. Similarly, if $48<k \leq 97$, then $x<97^{2} \leq 9409$, and if $34<k \leq 48$, then $x<48^{9 / 4}<6065$. But $p_{i+1}-p_{i} \leq 34$ for $p_{i}<9551$ by Lemma 2.6 .

Let $25<k \leq 34$. Then $132<26 \sqrt{26} \leq x<(34)^{5 / 2}<6741$. By (3.2) we have $P\left({ }_{k}(x)\right) \leq 5 k / 2 \leq 85$. Now we apply Lemma $2.5($ a).

Let $21 \leq k \leq 25$. Then $96<21 \sqrt{21} \leq x \leq 90$ by Lemma 2.5 (b).

For proceeding further, we need Lemma 3 of [25] which equals Corollary 4.2 .4 of [18].

Lemma 3.6. Let $2 k \leq x<k^{3 / 2}$ and assume (3.5). Then

$$
\left(\begin{array}{l}
x \\
k
\end{array}\right) \leq(2.83)^{k+\sqrt{x}} x^{k-\mu} .
$$

We observe that inequality (3) of [25] is not used in the proof of Lemma 3 of [25].

We apply the above result in the proof of the following lemma. 
Lemma 3.7. Let $x<k^{3 / 2}$ and $\rho=2$. Assume (3.2) and $l+s<1.05 k / \log k$. Then there exists an effectively computable absolute constant $k_{0}$ such that for $k>k_{0}$ we have $x<25 k$.

Proof. Put $\gamma=25$ and assume $x \geq \gamma k$. Then, by Lemma 3.6,

$$
\left(\begin{array}{c}
\gamma k \\
k
\end{array}\right) \leq\left(\begin{array}{l}
x \\
k
\end{array}\right) \leq(2.83)^{k+\sqrt{x}} x^{k-\mu}
$$

Now, by Lemma 2.11,

$$
\left(\begin{array}{c}
\gamma k \\
k
\end{array}\right)=\frac{(\gamma k) !}{k !((\gamma-1) k) !}>\frac{1}{\sqrt{2 \pi k}}\left(\frac{\gamma^{\gamma}}{(\gamma-1)^{\gamma-1}}\right)^{k} e^{-\frac{\gamma}{\gamma-1} \frac{1}{12 k}} .
$$

Let $k \geq k_{1}$ where $k_{1}$ is a sufficiently large effectively computable number depending only on $\gamma$. Then

$$
e^{-\frac{\gamma}{\gamma-1} \frac{1}{12 k}}>\frac{999}{1000}
$$

and

$$
\frac{\gamma^{\gamma}}{(\gamma-1)^{\gamma-1}}=(\gamma-1)\left(1+\frac{1}{\gamma-1}\right)^{\gamma} \geq e(\gamma-1)
$$

Thus

$$
\left(\begin{array}{c}
\gamma k \\
k
\end{array}\right)>\frac{999}{1000} \frac{1}{\sqrt{2 \pi k}}(e(\gamma-1))^{k} .
$$

On the other hand, by $m=2 k,(3.8),(3.4)$ and Lemma 2.10 ,

$$
\begin{aligned}
\left(\begin{array}{c}
\gamma k \\
k
\end{array}\right) & \leq(2.83)^{k+k^{3 / 4}} k^{\frac{3}{2}(\pi(2 k)-\pi(k)+l+s-1)} \\
& \leq(2.83)^{k+k^{3 / 4}} k^{\frac{3 k}{\log (2 k)}\left(1+\frac{1.2762}{\log (2 k)}\right)-\frac{3 k}{2 \log k-1}+\frac{3}{2}(l+s-1)}<e^{4.116 k} .
\end{aligned}
$$

By combining upper and lower estimates for $\left(\begin{array}{c}\gamma k \\ k\end{array}\right)$, we have

$$
(e(\gamma-1))^{k}<\frac{1000}{999} \sqrt{2 \pi k} e^{4.116 k}<e^{4.12 k} .
$$

Thus $24=\gamma-1<e^{3.12}<24$, a contradiction.

LEMmA 3.8. Let $l=1, s=0, \rho=5 / 2, m=k \rho$ and $x<k^{3 / 2}$. Then (3.2) implies that $x<12 k$.

Proof. Let $k \geq 1000$ and $x \geq 16 k$. Put $\gamma=16$. Then (3.8) and (3.9) are valid. Further we observe that

$$
e^{-\frac{\gamma}{\gamma-1} \frac{1}{12 k}}>.999
$$

Thus, by (3.9) and (3.10),

$$
\left(\begin{array}{c}
\gamma k \\
k
\end{array}\right) \geq .999 \frac{1}{\sqrt{2 \pi k}}(e(\gamma-1))^{k}
$$


On the other hand, as in the proof of Lemma 3.7, we have

$$
\left(\begin{array}{c}
\gamma k \\
k
\end{array}\right) \leq(2.83)^{k+k^{0.75}} k^{\frac{3.75 k}{\log (2.5 k)}\left(1+\frac{1.2762}{\log (2.5 k)}\right)-\frac{3 k}{2 \log k}} .
$$

This combined with (3.11) implies $k<1000$.

Let $12 k \leq x<16 k$. Put $\gamma=12$. Then (3.8), (3.9) and (3.11) are valid. On the other hand, as in the proof of Lemma 3.7, we have

$$
\left(\begin{array}{c}
\gamma k \\
k
\end{array}\right) \leq(2.83)^{k+4 \sqrt{k}}(16 k)^{\frac{2.5 k}{\log (2.5 k)}\left(1+\frac{1.2762}{\log (2.5 k)}\right)-\frac{k}{\log k}} .
$$

This combined with (3.11) implies $k<1000$. The lemma is trivial for $k \leq$ 144 , since $k^{3 / 2} \leq 12 k$ for such $k$. We use exact values of $\left(\begin{array}{c}\gamma k \\ k\end{array}\right)$ and the $\pi$ function in

$$
\left(\begin{array}{c}
\gamma k \\
k
\end{array}\right) \leq(2.83)^{k+k^{0.75}} k^{1.5(\pi(2.5 k)-\pi(k))}
$$

to show that this inequality is wrong for $144<k<1000$.

4. The basic results. First we prove Theorem 1.1 for $a \leq k$, which extends Schur's results for $a=0,1$. In fact his result for $a=0$ is used in the proof. We tacitly assume in Sections $4-7$ that $a \geq 0$.

Lemma 4.1. Let $a$ and $k$ be integers such that

$$
2 \leq k \leq n / 2, \quad 0 \leq a \leq k .
$$

Let $f(x)$ be given by (1.2) where $a_{0}, a_{1}, \ldots, a_{n}$ are integers satisfying (1.1). Assume that $f(x)$ has a factor of degree $k$. Then

$$
(n, k, a) \in\{(7,2,2),(7,3,3),(8,2,1),(8,3,2)\} .
$$

In this section we assume that the conditions of Theorem 1.1 hold. The proof of Lemma 4.1 depends on the following criterion for irreducibility.

Lemma 4.2. Assume that $f(x)$ has a factor of degree $k$. Suppose that there exists a prime $p>k+a$ such that $p$ divides ${ }_{k}(n+a)$. Then $p$ divides $a_{0} a_{n}$.

Corollary 4.3. Let $\left|a_{0}\right|=\left|a_{n}\right|=1$. Assume that $f(x)$ has a factor of degree $k$. Then $P(k(n+a)) \leq k+a$.

Proof of Lemma 4.2. Suppose that ${ }_{k}(n+a)$ is divisible by a prime $p$ with $p>k+a$ and $p \nmid a_{0} a_{n}$. Consider the Newton function $G_{p}(x)$ with respect to $p$ of

$$
G(x):=\sum_{j=0}^{n} b_{j} x^{j}:=(n+a) ! \sum_{j=0}^{n} \frac{x^{j}}{(j+a) !} \quad \text { with } \quad b_{j}=\frac{(n+a) !}{(j+a) !} .
$$


On the one hand $\left.p\right|_{k}(n+a)$, hence $p \mid b_{j}=\frac{(n+a) !}{(j+a) !}$ for $j \leq n-k$. So, by Corollary 2.14, it remains to show that the slope $\phi$ of the rightmost edge of the Newton function of $G_{p}$ is $<1 / k$. Denoting $\nu_{p}$ by $\nu$ we have

$$
\phi=\max _{1 \leq j \leq n} \frac{\nu\left(b_{0}\right)-\nu\left(b_{j}\right)}{j} \leq \max _{1 \leq j \leq n} \frac{\nu((j+a) !)}{j} .
$$

We may assume $p \leq j+a$, otherwise $\nu((j+a) !)=0$. Thus $j \geq p-a$ and

$$
\phi<\max _{j \geq p-a} \frac{j+a}{j(p-1)}=\frac{p}{p-1} \frac{1}{p-a} \leq \frac{k+1}{k} \frac{1}{k+1}=\frac{1}{k} .
$$

By Corollary 2.14 the polynomial $G(x)$ cannot have a factor of degree $k$.

Proof of Lemma 4.1. Let $2 \leq k \leq n / 2$ and $(n, k, a) \neq(8,2,1)$. We assume that $f(x)$ has a factor of degree $k$. Then $a \geq 1$ by the result of Schur [26]. If $(n, k)=(8,2)$, then $a=2$, which is not possible by Corollary 2.14 with $p=5$. From now on $(n, k) \neq(8,2)$.

First, we suppose that $a \leq .5 k$. By Corollary 4.3 , we see that

$$
P\left({ }_{k}(n+a)\right) \leq k+a \leq 1.5 k,
$$

which by Lemma 2.2 implies that $(n+a, k)=(4,2),(9,2),(10,5)$. Further we observe that $a=1$ if $k=2$ and $a \in\{1,2\}$ if $k=5$. Then $(n, k) \in$ $\{(3,2),(8,5),(9,5)\}$, which is ruled out since $n \geq 2 k$. Thus $a>.5 k$, implying $a \geq 2$.

Let $.5 k<a \leq .8 k$. Then $k \geq 3$. Further we see from Corollary 4.3 that $P(k(n+a)) \leq k+a \leq 1.8 k$. Now we apply again Lemma 2.2 with $x=n+a$ to conclude that

$$
(n+a, k) \in\{(10,3),(10,4),(28,13)\}
$$

since $x=n+a \geq 2 k+2$. In all cases but $(10,3)$ we have $k \geq 4$ and $n+a \leq 2 k+2$ and therefore

$$
k \leq n / 2 \leq k+1-a / 2<3 k / 4+1,
$$

a contradiction. If $(n+a, k)=(10,3)$, then $a=2$, hence $(n, k, a)=(8,3,2)$. Thus we conclude that $a>.8 k$.

Assume that $.8 k \geq 12$. Then

$$
n+a \geq \max \left(2 k+13, \frac{541}{262} k\right) .
$$

Now we derive from Lemma 2.4 that $P\left({ }_{k}(n+a)\right)>2 k \geq k+a$ contradicting Corollary 4.3 . Thus $.8 k<12$, i.e. $k<15$.

Let $.8 k<a \leq .95 k$. By Corollary 4.3 , we see that

$$
P\left({ }_{k}(n+a)\right) \leq k+a \leq 1.95 k .
$$

Now we apply Lemma 2.3 to conclude that

$$
n+a \in\{2 k, \ldots, 2 k+h-1\}
$$


with $k \in E_{h}$ and $1 \leq h \leq 5$. Let $h \in\{4,5\}$. Then $k \geq 12$ and $n+a=2 k+j$ with $0 \leq j \leq 4$. On the other hand, $2 k \leq n=2 k+j-a<2 k+4-.8 k<2 k$ since $k \geq 12$. Let $h \in\{2,3\}$. Then $n+a=2 k+j$ with $0 \leq j \leq 2$ and $2 k \leq n \leq 2 k+2-.8 k<2 k$ since $k \geq 4$. Let $h=1$. Then $n+a=2 k+j$ with $j=0$ and $2 k \leq n \leq 2 k-.8 k<2 k$. Hence $.95 k<a \leq k$. Since $k<15$, we have $a=k$. Now we see from Corollary 4.3 that

$$
P\left({ }_{k}(n+k)\right) \leq 2 k .
$$

Let $7 \leq k \leq 14$. Then there are three consecutive terms in ${ }_{k}(n+k)$ divisible by primes $\leq 13$ and we derive from Lemma $2.1(\mathrm{~d})$ a contradiction with (4.2). Let $4 \leq k \leq 6$. Then, for $n+k>16$, we refer to Lemma 2.1(e) and use the fact that $P\left({ }_{4}(n+k)\right) \leq 12$. Hence we may assume $12 \leq n+k \leq 16$ implying $k \in\{4,5\}$ and we check that $P\left({ }_{k}(n+k)\right)>2 k$. Let $k=3$. Then $n=7$ by $n \geq 6$ and Lemma $2 \cdot 1(\mathrm{~d})$. Thus $(n, k, a)=(7,3,3)$. Let $k=2$. Since $a=k$, we see from (4.2) that $P((n+1)(n+2)) \leq k+a=4$ implying $n=7$. Thus $(n, k, a)=(7,2,2)$.

The following examples show that the exceptions in Lemma 4.1 are necessary. In fact, it is possible to derive congruence conditions on the $a_{i}$ 's to guarantee that there is a factor of the indicated degree. In this way one can give infinitely many examples for each triple.

$$
\begin{aligned}
& \frac{x^{7}}{9 !}+23 \frac{x^{6}}{8 !}-73 \frac{x^{5}}{7 !}+16 \frac{x^{2}}{4 !}+8 \frac{x}{3 !}+\frac{1}{2 !} \\
& =\frac{1}{9 !}\left(x^{2}-3 x-6\right)\left(x^{5}+210 x^{4}-4620 x^{3}-12600 x^{2}-65520 x-30240\right) \text {, } \\
& \frac{x^{7}}{10 !}+2 \frac{x^{6}}{9 !}+4 \frac{x^{5}}{8 !}+15 \frac{x^{4}}{7 !}+4 \frac{x^{3}}{6 !}-10 \frac{x^{2}}{5 !}+\frac{1}{3 !} \\
& =\frac{1}{10 !}\left(x^{3}+20 x^{2}-60 x-120\right)\left(x^{4}+420 x^{2}+2520 x-5040\right), \\
& \frac{x^{8}}{9 !}+3 \frac{x^{7}}{8 !}-\frac{x^{6}}{7 !}-2 \frac{x^{5}}{6 !}+\frac{x^{3}}{4 !}+\frac{x^{2}}{3 !}+\frac{x}{2 !}+\frac{1}{1 !} \\
& =\frac{1}{9 !}\left(x^{2}+6 x+12\right)\left(x^{6}+21 x^{5}-210 x^{4}+2520 x^{2}+30240\right) \text {, } \\
& \frac{x^{8}}{10 !}-104 \frac{x^{7}}{9 !}-121 \frac{x^{6}}{8 !}-56 \frac{x^{5}}{7 !}-15 \frac{x^{4}}{6 !}+6 \frac{x^{3}}{5 !}+6 \frac{x^{2}}{4 !}+4 \frac{x}{3 !}+\frac{1}{2 !} \\
& =\frac{1}{10 !}\left(x^{3}+10 x^{2}+30 x+60\right)\left(x^{5}-1050 x^{4}-420 x^{3}-2520 x^{2}+25200 x+30240\right) \text {. }
\end{aligned}
$$

We use Lemma 4.1 to derive an explicit result in case $0 \leq a \leq 30$. As already stated in Section 1 , the cases $a=0$ and $a=1$ are due to Schur [26], 27]. 
Theorem 4.4. Let $f(x)$ be given by (1.2) with (1.1) and $3 \leq k \leq n / 2$.

(a) If $0 \leq a \leq 30$ and $k \geq 5$, then $f(x)$ has no factor of degree $k$ unless

$$
(n, k, a) \in\{(17,5,11),(19,5,9),(40,5,12)\} .
$$

(b) If $0 \leq a \leq 10$ and $3 \leq k \leq 4$, then $f(x)$ has no factor of degree $k$ unless

$$
\begin{aligned}
&(n, k, a) \in\{(7,3,3),(8,3,2),(12,3,4), \\
&(18,4,9),(18,4,10),(46,3,4),(56,4,10)\} .
\end{aligned}
$$

Proof. Assume that $f(x)$ has a factor of degree $k$. By Lemma 4.1 and Corollary 4.3 we may assume $k<a$ and $P\left({ }_{k}(n+a)\right) \leq a+k$.

(a) Let $5 \leq k \leq 6$. Then $n \geq 10, n+a>15$ and $P\left({ }_{k}(n+a)\right) \leq 31$. By Lemma 2.1(a) this is only possible if $n+a \leq 36$ or $k=5, n+a \in$ $\{52,58,66,156\}$.

Let $23 \leq n+a<27$. Then $\left.23\right|_{k}(n+a)$, hence $a+k \geq 23$ and $a \geq 17$, contradicting that $n \geq 10, n+a<27$. Similarly contradictions are obtained for $19 \leq n+a<23,17 \leq n+a<19, n+a=16$.

For $34 \leq n+a \leq 36$ we find $\left.17\right|_{k}(n+a)$, hence $a+k \geq 17,10 \leq n \leq 25$ and eliminations by Corollary 2.14 with $p=17$ for $a \geq 17$, with $p=31$ if $k=6, a=16$ and with $p=11$ for the other values of $(n, k, a)$. For $29 \leq n+a<34$ we find $a=23, n=10$ and elimination by Corollary 2.14 with $p=29$. For $n+a=28$ we find $a+k \geq 13, n \leq 21$ and eliminations by Corollary 2.14 with $p=23$ for $k=6$, and for $k=5$ by Corollary 2.14 with $p=13$ for $10 \leq n \leq 15$ and with $p=7$ for $n=20,21$ and by Lemma 2.13 with $p=3, r=2$ for $n=16$ and $n=18$. For $n+a=27$ we find $a+k \geq 23$, $n=10, a=17, k=6$. This is eliminated by Corollary 2.14 with $p=13$.

Let $n+a=156$. Because 31 divides 155, we see that $a+k \geq$ $P\left({ }_{k}(n+a)\right) \geq 31$, and thus we need only consider $a$ 's with $a \geq 26$. The resulting cases $(n, a)=(126,30),(127,29),(128,28),(129,27),(130,26)$ can each be eliminated by Corollary 2.14 with $p=13$. For $n+a=66$ a similar reasoning gives $a+k \geq 31,36 \leq n \leq 40$ and eliminations by Corollary 2.14 with $p=13$. For $n+a=58$ we obtain $a+k \geq 29,28 \leq n \leq 34$ and eliminations by Corollary 2.14 with $p=19$. For $n+a=52$ we find $a+k \geq 17$, $22 \leq n \leq 40$. These cases are eliminated by Corollary 2.14 with $p=17$ for $24 \leq n \leq 35$, and with $p=13$ for the remaining values of $n$ except for $n=40$.

Let $7 \leq k \leq 10$. Then $n \geq 14, n+a>21$ and $P\left({ }_{k}(n+a)\right) \leq 37$. By Lemma 2.1(a) this is only possible if $n+a \leq 40$. Let $37 \leq n+a<41$. Then $a+k \geq 37$, hence $a \geq 27$, contradicting $n \geq 14$. Similarly contradictions are obtained for $29 \leq n+a<33,23 \leq n+a<27, n+a=22$. Let $33 \leq n+a<37$. Then $a+k \geq 31$, hence $a \geq 21$. This implies $n+a \in\{35,36\}$, $a \in\{21,22\}, k \geq 9$. These cases are eliminated by Corollary 2.14 with $p=17$. Let $27 \leq n+a<29$. Then $a+k \geq 23$, hence $a \geq 13$. This implies 
$a \in\{13,14\}, k \geq 9$. These cases are eliminated by Corollary 2.14 with $p=13$.

Let $k \in\{11,12\}$. Then $n+a>33$ and $P\left({ }_{k}(n+a)\right) \leq 41$. By Lemma 2.1(a) this implies $n+a \leq 42$. If $37 \leq n+a \leq 42$, then $a+k \geq 37$, hence $a \geq 25$ in contradiction with $n \geq 22$. A similar reasoning excludes $34 \leq n+a \leq 36$.

Let $13 \leq k \leq 16$. Then $n+a>39$ and $P(k(n+a)) \leq 43$ and we see that there are seven consecutive terms in ${ }_{k}(n+a)$ composed of primes $\leq 41$ or two pairs of six separated by an integer $x$ with $P(x)=43$ in between. By Lemma 2.1(a) the latter possibility is excluded and the former implies $n+a \leq 46$. The remaining possibilities lead to contradictions by using Corollary 2.14 with $p=37$.

Let $17 \leq k \leq 22$ and $(n, k, a) \neq(34,17,18)$. Then $n+a>51$ and $P\left({ }_{k}(n+a)\right) \leq 47$ and there are six consecutive terms in ${ }_{k}(n+a)$ with prime factors $\leq 41$ or three blocks of five prime factors $\leq 41$ such that consecutive blocks are separated by one integer. This is excluded by Lemma 2.1(a). The case $(n, k, a)=(34,17,18)$ is excluded by Corollary 2.14 with $p=47$.

Let $23 \leq k \leq 28$. Then $n+a>69$ and $P\left({ }_{k}(n+a)\right) \leq 53$ and the previous argument applies.

Let $29 \leq k \leq 30$. Then $n+a>87$ and $P\left({ }_{k}(n+a)\right) \leq 59$ and the previous argument applies again.

(b) Let $k \in\{3,4\}$ and $(n, k, a) \neq(7,3,3),(8,3,2)$. Then we see from Lemma 4.1 that $a \geq 4, n \geq 6$ if $k=3$ and $a \geq 5, n \geq 8$ if $k=4$.

Let $a \in\{4,5,6,7\}$ if $k=3$ and $a \in\{5,6\}$ if $k=4$. Then, by Corollary 4.3, $P\left({ }_{k}(n+a)\right) \leq 7$, which implies $k=3$ by Lemma 2.1(e). Further we see from Lemma 2.1(d) that

$$
(n, a) \in\{(6,4),(9,7),(10,6),(11,5),(12,4),(43,7),(44,6),(45,5),(46,4)\} .
$$

We observe that $(6,4)$ is excluded by Lemma 2.13 with $p=3, r=1$, $(n, a)=(10,6),(11,5),(44,6),(45,5)$ by Corollary 2.14 with $p=5$ and $(n, a)=(9,7),(43,7)$ with $p=7$.

Let $a \in\{8,9\}$ if $k=3$ and $a \in\{7,8\}$ if $k=4$. Then $P\left({ }_{k}(n+a)\right) \leq 11$. Then we derive from Lemma 2.1(d),(e) that $(n, a)$ is in

$$
\{(7,9),(8,8),(13,9),(14,8),(41,9),(42,8),(47,9),(48,8),(91,9),(92,8)\}
$$

with $k=3$. All these cases are excluded by Corollary 2.14 with $p=7$.

Let $k=4$ and $a \in\{9,10\}$. Then $P(k(n+a)) \leq 13$. We derive from Lemma 2.1(e) that

$$
(n, a) \in\{(17,10),(18,9),(18,10),(19,9),(56,10),(57,9)\} .
$$

The cases $(n, a)=(19,9),(57,9)$ are excluded by Corollary 2.14 with $p=7$, the case $(n, a)=(17,10)$ by Lemma 2.13 with $p=3, r=2$. 
Let $k=3$ and $a=10$. Then we derive from Lemma 2.1(d) that $n \in$ $\{6,12,16,17,18,40,46,55,56,90,342\}$. The case $n=18$ is excluded by Corollary 2.14 with $p=7$ and all others with $p=5$.

Proof of Theorem 1.1. Let the assumptions of Theorem 1.1 be satisfied and $(n, k, a)$ not in the set given in (1.3). Then

$$
k<a \leq 3 k / 2
$$

by Lemma 4.1 .

Let $k>2$. Then $k \geq 5$ by Theorem 4.4(b). Now we see from Theorem 4.4(a) that $a>30$ implying $k>20$. We put $x=n+a$. Thus $x>2 k+k=3 k>60$. Let $l=1, s=0, \rho=5 / 2, m=k \rho$. By Corollary 4.3 we have

$$
P\left({ }_{k}(x)\right) \leq 5 k / 2 .
$$

Consequently, $x<k^{3 / 2}$ by Lemma 3.5. We derive from Lemma 3.8 that $x<12 k$. Now we derive from Lemma 2.7 that

$$
P(k(x))>x-5 k / 6 \geq 3 k-5 k / 6=13 k / 6 .
$$

Now, by Corollary 4.3, we see that $a>\frac{7}{6} k$, implying that $x>\frac{19}{6} k$. Another application of Lemma 2.7 gives $P(k(x))>\frac{7}{3} k$ whence $a>\frac{4}{3} k, x>\frac{10}{3} k$, $P\left({ }_{k}(x)\right)>2.5 k$, contradicting (4.3).

Let $k=2$. Then $a=3, n+a \geq 7$ and $P\left({ }_{2}(n+a)\right) \leq 5$ by Corollary 4.3. Now we apply Lemma 2.1(f) to obtain $n \in\{6,7,13,22,78\}$.

REMARK. Examples of factorizations for $(6,2,3)$, $(7,2,3),(12,3,4)$, $(13,2,3),(22,2,3),(46,3,4)$, and $(78,2,3)$ are as follows:

$$
\frac{x^{6}}{9 !}+4 \frac{x^{5}}{8 !}-6 \frac{x^{4}}{7 !}+\frac{x^{3}}{6 !}-\frac{x^{2}}{5 !}+2 \frac{x}{4 !}+\frac{1}{3 !}
$$

is divisible by $x^{2}-6 x+12$,

$$
\frac{x^{7}}{10 !}+10 \frac{x^{6}}{9 !}+24 \frac{x^{5}}{8 !}+7 \frac{x^{4}}{7 !}+5 \frac{x^{3}}{6 !}+2 \frac{x^{2}}{5 !}+2 \frac{x}{4 !}+\frac{1}{3 !}
$$

is divisible by $x^{2}+30 x+60$,

$$
\frac{x^{12}}{16 !}-107 \frac{x^{9}}{13 !}-45 \frac{x^{6}}{10 !}+9 \frac{x^{3}}{7 !}+\frac{1}{4 !}
$$

is divisible by $x^{3}+840$,

$$
\begin{aligned}
\frac{x^{13}}{16 !}-\frac{x^{12}}{15 !}+44 \frac{x^{11}}{14 !}-9 & \frac{x^{10}}{13 !}+2 \frac{x^{9}}{12 !}+15 \frac{x^{8}}{11 !}-2 \frac{x^{7}}{10 !} \\
& +\frac{x^{6}}{9 !}-8 \frac{x^{5}}{8 !}+5 \frac{x^{4}}{7 !}-12 \frac{x^{3}}{6 !}+2 \frac{x^{2}}{5 !}-\frac{x}{4 !}+\frac{1}{3 !}
\end{aligned}
$$


is divisible by $x^{2}-30 x+60$,

$$
\frac{x^{22}}{25 !}+76 \frac{x^{20}}{23 !}+9 \frac{x^{18}}{21 !}+\frac{x^{6}}{9 !}+2 \frac{x^{4}}{7 !}+2 \frac{x^{2}}{5 !}+\frac{1}{3 !}
$$

is divisible by $x^{2}+60$,

$$
\begin{array}{rl}
\frac{x^{46}}{50 !}+1554183979460315 & 52910267363 \frac{x^{43}}{47 !} \\
+ & 1287 \frac{x^{12}}{16 !}+25118061500 \frac{x^{4}}{8 !}+184 \frac{x^{3}}{7 !}+\frac{1}{4 !}
\end{array}
$$

is divisible by $x^{3}+840$. For the triple $(n, k, a)=(78,2,3)$, one can use

$$
\begin{aligned}
& \frac{x^{78}}{81 !}-699547017649181565404859682868563695329144745169587091 \frac{x^{76}}{79 !} \\
& +\frac{x^{62}}{65 !}+3820 \frac{x^{58}}{61 !}+15 \frac{x^{54}}{57 !}+\frac{x^{46}}{49 !}+813385 \frac{x^{12}}{15 !}+22 \frac{x^{4}}{7 !}+2 \frac{x^{2}}{5 !}+\frac{1}{3 !},
\end{aligned}
$$

which has the factor $x^{2}+60$.

Factorizations for $(7,2,2),(7,3,3),(8,2,1),(8,3,2)$ have been given after the proof of Lemma 4.1.

5. A better estimate for $a$ when $k$ is sufficiently large. We prove

Theorem 5.1. Let $f(x)$ be given by (1.2). Let $n \geq 1,1 \leq k \leq n / 2$ and $\left|a_{0}\right|=\left|a_{n}\right|=1$. Assume that $f(x)$ has a factor of degree $k$. Then there exist effectively computable absolute constants $k_{0}$ and $C>0$ such that for $k \geq k_{0}$, we have

$$
a \geq C k \log k \frac{\log \log k}{\log \log \log k} .
$$

Proof. Let $k$ be sufficiently large. We show that there exists a prime $p>k+a$ dividing ${ }_{k}(n+a)$. Let $n \leq k^{3 / 2}$. Then the interval $[n+a-k+1$, $n+a]$ contains primes and each prime is at least $n+a-k+1>k+a$. The existence of the primes follows from a well-known result on differences between consecutive primes [17]. Thus we may suppose that $n>k^{3 / 2}$. Then we see from [30] that $P\left({ }_{k}(n+a)\right)$ is at least constant times $k \log k \frac{\log \log k}{\log \log \log k}$. Hence there is a positive constant $C$ such that $P(k(n+a))>k+a$ whenever $a<C k \log k \frac{\log \log k}{\log \log \log k}$. Then, by Corollary 4.3, $f(x)$ has no factor of degree $k$, a contradiction. Hence we conclude that

$$
a \geq C k \log k \frac{\log \log k}{\log \log \log k} .
$$

6. The leading coefficient is a prime power. In the next two sections we shall relax the condition that both $a_{0}$ and $a_{n}$ are \pm 1 . In this section we prove Theorem 1.2. 
Lemma 6.1. Let $f(x)$ be given by (1.2). Let $n \geq 1,2 \leq k \leq n / 2,0 \leq a$ $\leq .5 k$ and $\omega\left(a_{n} a_{0}\right)=1$. Assume that $f(x)$ has a factor of degree $k$. Then $k \leq 5$ unless $(n, k, a)=(24,9,4), P\left(a_{0} a_{n}\right)=23$. If $3 \leq k \leq 5$, then $n+a$ $\leq 28$. If $k=2$, then $a=1$.

Proof. Let the assumptions of Lemma 6.1 be satisfied. We may suppose $\omega\left(a_{n}\right)=1,\left|a_{0}\right|=1$ since the proof of the case $\omega\left(a_{0}\right)=1,\left|a_{n}\right|=1$ is similar and the case $a_{n}=p^{r}, a_{0}=p^{s}$ can be reduced to it by multiplying by $p^{r n-r}$ and replacing $p^{r} x$ by $x$. Assume that $f(x)$ has a factor of degree $k$. We omit a term in ${ }_{k}(n+a)$ divisible by $P\left(a_{n}\right)$ and denote the remaining product by ${ }_{k}(n+a)^{(1)}$. Then, by the proof of Lemma 4.2 applied to any prime different from $P\left(a_{n}\right)$,

$$
P\left(k(n+a)^{(1)}\right) \leq k+a \leq 1.5 k .
$$

Now we derive from Theorem 2.12 that $k \leq 9$ or

$$
(n+a, k) \in\{(26,13),(27,13),(28,13),(28,14)\} .
$$

Let $(n+a, k) \in\{(26,13),(27,13),(28,13),(28,14)\}$. Then $a \leq 2$ because $n \geq 2 k$. Hence we find that there is no factor of degree $k$ by applying Corollary 2.14 with the primes $p=19$ and $p=23$.

Let $k=9$. Then $a \leq 4$. We may suppose by (6.1) that there is at most one term in ${ }_{k}(n+a)$ divisible by a prime greater than 13 and we omit this term. There is at most one term divisible by each of 13 and 11 and there are at most two terms divisible by 7 and we omit all of them. Thus we are left with a term $n^{*}$ composed of primes $\leq 5$, and for each of these primes, there is another term in which it appears to a power which is at least the power in $n^{*}$. Thus $n+a-8 \leq 8 \cdot 3 \cdot 5=120$. Thus $n+a \leq 128$ if $k=9$. Similarly $n+a \leq 67$ if $k=8, n+a \leq 18$ if $k=7$ and $n+a \leq 65$ if $k=6$. Further we check that for each of the above cases, ${ }_{k}(n+a)$ has two terms each divisible by a prime $>k+a$, contradicting (6.1). This follows by direct computation except for $(n, k, a)=(24,9,4)$ in which case we apply Corollary 2.14 with respect to the prime 23 . Thus $k \leq 5$.

Let $3 \leq k \leq 5$ and $n+a>28$. As above, we see that there are two terms in ${ }_{k}(n+a)$ composed of 2 and 3 in each of the cases $3 \leq k \leq 5$. On using the fact that all powers of 2 and 3 which differ by 1 are $\leq 9$ (cf. [22, Table 1A]) we derive that $n+a \leq 36$. By checking the possibilities we find that (6.1) is not satisfied. Consequently, $k=2$. If $a=0$, then either $n$ or $n-1$ is a power of 2 , and 2 does not divide $a_{n}$. These cases have been excluded in [9].

For integers $N>1$ and $k \geq 1$, we write $\omega_{k}(N)$ for the number of distinct prime divisors $>k$ of $N$. Further we put $\omega_{k}(1)=0$ and $\omega_{k}(N)=\omega_{k}(|N|)$ for any non-zero integer $N$. 
Proof of Theorem 1.2. By Lemma 6.1, we may suppose that $a>.5 k$. Also

$$
P\left({ }_{k}(n+a)^{(1)}\right) \leq k+a
$$

by Lemma 4.2 . Let $k \geq 19$. We observe that $n+a>2.5 k$. Further we derive from Lemma 2.9 when $n+a>k^{2}$ and Lemma 2.8(b) when $n+a \leq k^{2}$ that

$$
\omega_{k}\left(k_{k}(n+a)^{(1)}\right) \geq \pi(2 k)-\pi(k)
$$

and

$$
\omega_{k}\left(k(n+a)^{(1)}\right) \geq \pi(2 k)-\pi(k)-1,
$$

respectively. On the other hand, in view of (6.2) and $a<.75 k$, we may suppose that

$$
\omega_{k}\left({ }_{k}(n+a)^{(1)}\right) \leq \pi(1.75 k)-\pi(k) .
$$

Thus

$$
\pi(2 k)-\pi(1.75 k) \leq 0 \quad \text { if } n>k^{2}
$$

and

$$
\pi(2 k)-\pi(1.75 k) \leq 1 \quad \text { if } n \leq k^{2}
$$

whence, by Lemma 2.10 for $k \geq 400$ and direct calculation for $65<k<400$,

$$
k \leq 65 .
$$

Hence $P_{k}\left((n+a)^{(1)}\right) \leq 1.75 k \leq 114$.

Let $24 \leq k \leq 65$. Then there are 12 consecutive integers composed of primes at most 114. According to Lemma 2.15 this is only possible if $n+a$ $\leq 330$. But such triples $(n, k, a)$ do not satisfy (6.2) in view of Lemma 2.6.

Let $21 \leq k \leq 24$. Then $\pi(2 k)-\pi(1.75 k)>1$, contradicting (6.3) and (6.4).

Let $13 \leq k \leq 20$. Then $n+a \geq 33, k+a \leq 35$ and there are six consecutive terms with prime factors $\leq 31$, which possibility is excluded by Lemma 2.1(a).

Let $10 \leq k \leq 12$. Then $n+a>25, k+a \leq 21$ and there are six consecutive terms composed of primes $\leq 19$ or one block of four and one block of five such terms separated by one integer. This is also excluded by Lemma 2.1(a) unless $n+a=57$ in which case (6.2) is not satisfied.

Let $k=8$ or 9 . Then $n+a>20$ and we see from (6.2) and $a \leq .75 k$ that there are four consecutive terms in ${ }_{k}(n+a)$ divisible by primes at most 11 or 13 according as $k=8$ or 9 , respectively. Now we derive from Lemma $2.1(\mathrm{e})$ that $k=9$ and that they are given by ${ }_{4}(27),{ }_{4}(28)$ and ${ }_{4}(66)$, and these are excluded by $(6.2)$ unless $(n+a, k)=(28,9)$. This gives the exceptions $(n, k, a)=(22,9,6)$ and $(23,9,5)$ where $P\left(a_{0} a_{n}\right)=23$ in view of Corollary 2.14 with $p=23$. 
Let $k=6$ or 7 . Then $n+a>15$ and there are three consecutive terms in ${ }_{k}(n+a)$ composed of primes not exceeding 7 or 11 according as $k=6$ or 7 . Next we see from Lemma 2.1 (d) that they are given by ${ }_{3}(16),{ }_{3}(50)$ if $k \in\{6,7\}$ and moreover ${ }_{3}(22),{ }_{3}(56),{ }_{3}(100)$ if $k=7$. By $(6.2), a \leq .75 k$ and $n+a>2.5 k$ are all excluded.

Let $k=5$ and $n+a>32$. Then $a=3, n \geq 30$. Further there is at most one term divisible by a prime $p_{0}>7$. Suppose that $p_{0} \nmid n+a-2$. Then there are three consecutive terms divisible by primes $\leq 7$ and we derive from Lemma 2.1(d) that $n \leq 49$. By (6.2) we find that there are no exceptions. Assume that $p_{0} \mid n+a-2$. In that case, we see that

$$
P((n+a)(n+a-1)) \leq 7, \quad P((n+a-3)(n+a-4)) \leq 7 .
$$

Now we apply Lemma 2.1(f) and we see that the above inequalities do not hold.

Let $k=4$ and $n+a>32$. Then $a=3$ and $n \geq 30$. In this case, there are two consecutive terms divisible by primes $\leq 7$ and another such number at distance at most 2. By Lemma 2.1(f),(g) this is possible only if $n \in\{47,48,125\}$.

Let $k=3$ and $n+a>32$. Then $a=2$ and $n \geq 31$. Now there are either two consecutive terms or two terms differing by 2 divisible by primes $\leq 5$. We again apply Lemma 2.1(f), (g) to conclude that $n \in\{79,80\}$ in the first possibility and $n \in\{48,160\}$ in the second. The cases $n=48,79,80$ are mentioned as exceptions in Theorem 1.2. The case $n=160$ is excluded by Corollary 2.14 for the primes 7 and 23 .

Let $k=2$. Then there is no integer in $(k / 2,3 k / 4]$.

\section{The leading coefficient is divisible by more than one prime}

Theorem 7.1. Let $\epsilon>0,1 \leq k \leq n / 2,0 \leq a \leq(1-\epsilon) k$ and $f(x)$ be given by

$$
f(x)=a_{n} \frac{x^{n}}{(n+a) !}+a_{n-1} \frac{x^{n-1}}{(n-1+a) !}+\cdots+a_{1} \frac{x}{(1+a) !}+a_{0} \frac{1}{a !}
$$

where $a_{0}, a_{1}, \ldots, a_{n}$ are integers with $a_{n} \neq 0$ and $\left|a_{0}\right|=1$. Then there exist effectively computable numbers $c_{1}>0$ and $k_{0}$ depending only on $\epsilon$ such that for $k \geq k_{0}$, the polynomial $f(x)$ has no factor of degree $k$ whenever

$$
\omega_{k}\left(a_{n}\right)<c_{1} \frac{k}{\log k} \text {. }
$$

Proof. Let $\epsilon>0$ and $\omega_{k}\left(a_{n}\right)<k / \log k$. We may suppose that $k \geq k_{0}$ with $k_{0}$ sufficiently large. By Lemma 4.2 it suffices to find a prime $p>k+a$ satisfying

$$
\left.p\right|_{k}(n+a), \quad p \nmid a_{n} .
$$


Let $n \leq 25 k$. We consider the interval $(n+a-\epsilon k, n+a]$. By the prime number theorem, the above interval contains $c_{2} k / \log k$ primes where $c_{2}>0$ is an effectively computable number depending only on $\epsilon$. Each of the primes divides ${ }_{k}(n+a)$ and is greater than $n+a-\epsilon k \geq(2-\epsilon) k \geq k+a$, since $a \leq(1-\epsilon) k$. If we choose $c_{1}=c_{2} / 2$, then there exists a prime $p>k+a$ satisfying (7.1).

Next we consider $n>25 k$. For each prime $p>k$ dividing $a_{n}$ we take the unique term from ${ }_{k}(n+a)$ divisible by $p$ if it exists and we omit it. The number of these omitted terms is less than $1.04 k / \log k$. Now we apply Lemma 3.7 and Corollary 3.4 with $l=1, m=2 k, s<1.04 k / \log k, x=n+a$ if $n+a<k^{3 / 2}$ and if $n+a \geq k^{3 / 2}$, respectively, to conclude that (3.2) does not hold. Therefore there exists a prime $p>2 k \geq k+a$ satisfying (7.1).

Thus we see from Theorem 7.1 that there exists an effectively computable number $c_{3}>1$ depending only on $\epsilon$ such that $\left|a_{n}\right|>c_{3}^{k}$ whenever $f(x)$ has a factor of degree $k$.

8. Generalized Laguerre polynomials with $\alpha$ non-negative. We consider polynomials

$$
g_{a, b, c}(x)=\sum_{j=0}^{n} \frac{(a)_{j}}{(b)_{j}(c)_{j}} x^{j}
$$

where

$$
a=-n-s, \quad b=\alpha+1, \quad c \geq 1 .
$$

For $s=0, c=1$ this is the classical Laguerre polynomial. We prove Theorem 1.3 stated in the introduction.

Proof of Theorem 1.3. We may assume that $k \geq k_{1}=k_{1}(\epsilon)$ with $k_{1}$ sufficiently large. Put

$$
\begin{aligned}
h(x) & =(n+s+c-1) ! \sum_{j=0}^{n} \frac{(n+\alpha) \cdots(j+1+\alpha)}{(n+s-j) !(c+j-1) !} x^{j} \\
& =\sum_{j=0}^{n}\left(\begin{array}{c}
n+s+c-1 \\
c+j-1
\end{array}\right)_{n-j}(n+\alpha) x^{j} .
\end{aligned}
$$

Since

$$
g_{-n-s, \alpha+1, c}(x)=\frac{(c-1) !(n+s) !}{n(n+\alpha)} \sum_{j=0}^{n} \frac{(n+\alpha) \cdots(j+1+\alpha)}{(n+s-j) !(c+j-1) !}(-x)^{j},
$$

we observe that $h(x)$ has a factor of degree $k$ if and only if $g(x)$ has a factor of degree $k$. Therefore it suffices to prove Theorem 1.3 with $g(x)$ replaced 
by $h(x)$. For $0 \leq j \leq n$, we put

$$
a_{j}=\left(\begin{array}{c}
n+s+c-1 \\
c+j-1
\end{array}\right) .
$$

Then

$$
\begin{aligned}
& a_{n}=\left(\begin{array}{c}
n+s+c-1 \\
n+c-1
\end{array}\right)=\frac{(n+c) \cdots(n+c+s-1)}{s !}, \\
& a_{0}=\left(\begin{array}{c}
n+s+c-1 \\
c-1
\end{array}\right)=\frac{(n+s+1) \cdots(n+s+c-1)}{(c-1) !} .
\end{aligned}
$$

Because of Lemma 4.2 it suffices to show that there exists a prime $p>k+\alpha$ with $\left.p\right|_{k}(n+\alpha)$ such that

$$
p \nmid(n+c) \cdots(n+c+s-1), \quad p \nmid(n+s+1) \cdots(n+s+c-1) .
$$

Let $n<k^{3 / 2}$ and $\alpha<.5 k$. We consider the interval $(n-(.6 k-\alpha-1), n]$. It contains a prime $p>n+\alpha-.6 k+1 \geq 1.4 k+\alpha$. Assume that $p$ divides $(n+c) \cdots(n+c+s-1)$. Then

$$
n-j \equiv 0(\bmod p), \quad n+i \equiv 0(\bmod p)
$$

for some $i, j$ with $0 \leq j \leq .6 k, c \leq i<c+s$. Thus $i+j \equiv 0(\bmod p)$, implying $1.4 k+\alpha<i+j<.6 k+c+s$, contradicting (1.6). Consequently $p \nmid(n+c)_{s}$. Similarly $p \nmid(n+s+1)_{c-1}$. Next we consider $n<k^{3 / 2}, \alpha \geq .5 k$. Then we take the interval $(n+c+s, n+\alpha]$. By (1.6) and [17] we see that it contains a prime $p>n+c+s \geq 2 k \geq k+\alpha$. By a similar reasoning we deduce that $p$ satisfies (8.1).

Let $n \geq k^{3 / 2}$. We omit all the terms $n+c, \ldots, n+c+s-1$ and $n+s+1$, $\ldots, n+s+c-1$ from $n+\alpha-k+1, \ldots, n+\alpha$. The number of omitted terms is at most $v \leq s+c$. Now we apply (1.6) and Corollary 3.4 with $x=n+\alpha$, $s=v$ and $l=1$ to conclude that (3.2) does not hold. Therefore we find a prime $p>2 k \geq k+\alpha$ dividing $_{k}(n+\alpha)^{(v)}$. Assume that (8.1) is not satisfied. Then

$$
n-j \equiv 0(\bmod p), \quad n+i \equiv 0(\bmod p)
$$

with $-\alpha \leq j \leq k$ and $0<i \leq s+c$. It follows from the construction that $i+j \neq 0$. This implies that

$$
2 k<p \leq|i+j| \leq s+c+k<4 k / 3,
$$

a contradiction.

\section{Generalized Laguerre polynomials with $\alpha$ negative}

Proof of Theorem 1.4. In view of results of Schur [26], Hajir [15] and Sell [29], already mentioned in the introduction, we may assume without loss of generality that $s>2$, hence $k>3$. 
It suffices to show that the polynomial

$$
g(x):=\sum_{j=0}^{n} \frac{(n+s-j) ! n !}{(n-j) ! j ! s !} x^{j}=n ! \sum_{j=0}^{n}\left(\begin{array}{c}
n+s-j \\
n-j
\end{array}\right) \frac{x^{j}}{j !}
$$

has no factor of degree $k$. For $0 \leq j \leq n$, we put

$$
a_{j}=\left(\begin{array}{c}
n+s-j \\
n-j
\end{array}\right) \text {. }
$$

Then

$$
a_{n}=1, \quad a_{0}=\left(\begin{array}{c}
n+s \\
n
\end{array}\right) .
$$

By Lemma 4.2 it suffices to show that there exists a prime $p>k$ such that $\left.p\right|_{k}(n)$ and $p \nmid{ }_{s}(n+s)$. We derive from Lemma 2.3 that, unless $k \leq 270$ and $n \leq 2 k+10$, there exists a prime $p>1.95 k$ dividing ${ }_{k}(n)$. Assume that this prime divides ${ }_{s}(n+s)$. Then $n-j \equiv 0(\bmod p), n+i \equiv 0(\bmod p)$ with $0 \leq j<k, 0<i \leq s$, implying $1.95 k<p \leq i+j<s+k$. For each $k \leq 270$ we consider $n \in[2 k, 2 k+10]$ and $q$ to be the greatest prime $\leq n$.

We shall check that $q>n-k, 2 q>n+0.95 k$ unless $(n, k)=(10,5)$. For this, we may assume that $k>10$, otherwise the assertion follows by direct computation. Then $2 k \leq n \leq 2 k+10<2.95 k$ implying $n-k>(n+0.95 k) / 2$. Now the assertion follows from Lemma 2.6 and $n \geq 2 k$ by observing that the interval $((n+0.95 k) / 2, n]$ contains a prime. Finally we check that the polynomial $g_{a, b, c}$ is irreducible for $(n, s) \in\{(10,3),(10,4)\}$.

10. The rational case. Let $u, \alpha, \beta$ and $a$ be as in the paragraphs preceding Theorem 1.5 in Section 1. Let

$$
G(x)=a_{n} \frac{x^{n}}{(\alpha)_{\beta, n+u}}+a_{n-1} \frac{x^{n-1}}{(\alpha)_{\beta, n-1+u}}+\cdots+a_{1} \frac{x}{(\alpha)_{\beta, 1+u}}+a_{0} \frac{1}{(\alpha)_{\beta, u}}
$$

where $a_{0}, a_{1}, \ldots, a_{n} \in \mathbb{Z}$. Here $F(x)=G(\beta x)$. We put

$$
G_{1}(x)=(\alpha)_{\beta, n+u} G(x) .
$$

Thus

$$
\begin{aligned}
G_{1}(x)= & a_{n} x^{n}+a_{n-1}(\alpha+(n+u-1) \beta) x^{n-1}+\cdots \\
& +a_{1}(\alpha+(n+u-1) \beta) \cdots(\alpha+(u+1) \beta) x \\
& +a_{0}(\alpha+(n+u-1) \beta) \cdots(\alpha+u \beta) .
\end{aligned}
$$

We begin with the following result.

Lemma 10.1. Let $1 \leq k \leq n / 2$ and

$$
\beta \leq 2 \alpha+2 \quad \text { if }(k, u)=(1,0) .
$$


Assume that the prime $p$ satisfies

$$
p \geq \begin{cases}(k+u-1) \beta+\alpha+1 & \text { if } u>0, \\ (k+u-1) \beta+\alpha+2 & \text { if } u=0,\end{cases}
$$

and

$$
p \mid(\alpha+(n+u-k) \beta) \cdots(\alpha+(n+u-1) \beta), \quad p \nmid a_{0} a_{n} .
$$

Then $G(x)$ has no factor of degree $k$.

Proof. If suffices to show that $G_{1}(x)$ has no factor of degree $k$. Denote $\nu_{p}$ by $\nu$. We consider the Newton function $H(x)$ with respect to $p$ of the polynomial $h(x)$ obtained from $G_{1}(x)$ by putting $a_{0}=a_{1}=\cdots=a_{n}=1$. By Corollary 2.14 and $p \mid(\alpha+(n+u-k) \beta)_{\beta, k}$ it suffices to show that the slope $\phi$ of its rightmost edge is less than $1 / k$. We have

$$
\begin{aligned}
\phi & =\max _{1 \leq j \leq n} \frac{\nu\left((\alpha+u \beta)_{\beta, n}\right)-\nu\left((\alpha+(j+u) \beta)_{\beta, n-j}\right)}{j} \\
& =\max _{1 \leq j \leq n} \frac{\nu((\alpha+u \beta) \cdots(\alpha+(j+u-1) \beta))}{j} \\
& \leq \max _{1 \leq j \leq n} \frac{\nu((\alpha+(j+u-1) \beta) !)}{j} .
\end{aligned}
$$

Now

$$
\nu((\alpha+(j+u-1) \beta) !)<\frac{\alpha+(j+u-1) \beta}{p-1} .
$$

Thus

$$
\phi<\frac{\alpha+(j+u-1) \beta}{(p-1) j}=\frac{\alpha+(u-1) \beta}{(p-1) j}+\frac{\beta}{p-1} .
$$

We may assume that $p \leq \alpha+(j+u-1) \beta$, otherwise $\nu((\alpha+(j+u-1) \beta) !)=0$. Thus

$$
j \geq \frac{p-\alpha-(u-1) \beta}{\beta}
$$

and

$$
\phi<\frac{\alpha \beta+(u-1) \beta^{2}}{(p-1)(p-\alpha-(u-1) \beta)}+\frac{\beta}{p-1}=\frac{\beta p}{(p-1)(p-\alpha-(u-1) \beta)} .
$$

It suffices to show that

$$
\frac{\beta p}{(p-1)(p-\alpha-(u-1) \beta)} \leq \frac{1}{k}
$$

i.e.,

$$
p^{2}-((k+u-1) \beta+\alpha+1) p+\alpha+(u-1) \beta \geq 0,
$$

which is satisfied by (10.2) and (10.1).

It has been proved in [31, 32] that for $\chi=\alpha+(n+u-1) \beta$ and $\epsilon>0$, there exists an effectively computable number $C>0$ depending only on $\epsilon$ 
such that

$$
P:=P\left((\alpha+(n+u-k) \beta)_{\beta, k}\right)>C k \log \log \chi \quad \text { if } \chi>k(\log k)^{\epsilon} .
$$

Recall that the height $H(a)$ of a non-zero rational number $a$, in its reduced form, is defined as the maximum of the absolute values of its numerator and denominator. We write $N(a)$ for the absolute value of the numerator of $a$ and $D(a)$ for the denominator of $a$. Now we combine Lemma 10.1 and (10.3) with the prime number theorem for arithmetic progressions to prove the following result which implies Theorem 1.5 immediately.

THEOREM 10.2. Let $\left|a_{n}\right|=1,2 \leq k \leq n / 2$ and assume that $G(x)$ has a factor of degree $k$. Then there exist effectively computable absolute constants $k_{0}, C_{1}>0$ and $C_{2}$ such that for $k \geq k_{0}$ and $P\left(a_{0}\right)<C_{2} k / \log \log k$, we have

$$
H(a) \geq C_{1} \log \log k .
$$

Proof. In fact we shall prove the more precise assertion that there exist effectively computable absolute constants $C_{3}>0$ and $C_{4}>0$ such that for $k \geq k_{0}$,

$$
N(a) \geq C_{3} k \log \log k \quad \text { whenever } \quad D(a) \leq C_{4} \log \log k .
$$

Assume that $G(x)$ has a factor of degree $k$. Put $\chi=\alpha+(n+u-1) \beta$ and suppose that

$$
\chi>k \log k \text {. }
$$

Then we see from (10.3) with $\epsilon=1$ that

$$
P>C_{5} k \log \log k
$$

where $C_{5}>0$ is an effectively computable absolute constant. Now we choose $C_{2}<C_{5}$ and apply Lemma 10.1 and (10.2) to conclude that

$$
P \leq(k+u-1) \beta+\alpha+2 .
$$

The assertion follows by combining the above lower and upper bound for $P$ and choosing $C_{3}+C_{4}<C_{5} / 2$.

Thus we may suppose that

$$
\chi \leq k \log k .
$$

We observe that

$$
\alpha+(n+u-k) \beta \geq(k+u-1) \beta+\alpha+\beta,
$$

and that $\beta \geq 2$. Therefore, in view of Lemma 10.1, it suffices to show that there is a prime among

$$
\alpha+(n+u-k) \beta, \ldots, \alpha+(n+u-1) \beta
$$

which does not divide $a_{0}$. By the prime number theorem for arithmetic progressions with error term and (10.4), we see from [4, pp. 132-133] that the number of primes among (10.5) is at least $3 C_{2} k /(\log k \log \log k)$ if $C_{2}$ is 
sufficiently small and $k$ exceeds an effectively computable absolute constant. Therefore we can find a prime in (10.5) which does not divide $a_{0}$ since $\omega\left(a_{0}\right)<2 C_{2} k /(\log k \log \log k)$. We observe from [4, p. 133] that we have used the version of the prime number theorem for arithmetic progressions where the constant implied in the error term is effective and uniform with respect to $\beta$ since $\beta \leq C_{4} \log \log k$.

Corollary 10.3. Let $\left|a_{n}\right|=1$, and suppose that $P\left(a_{0}\right)$ is bounded. Fix $u, \alpha$ and $\beta$. Then there is an $n_{0}=n_{0}\left(u, \alpha, \beta, P\left(a_{0}\right)\right)$ such that if $n \geq n_{0}$, then $G(x)$ is irreducible or $G(x)$ has a linear factor, and a linear factor can only occur if $k=1, u=0$, and $\beta>2 \alpha+2$.

Proof. By Theorem 10.2 and $a=u+\alpha / \beta$, we see that $k$ is bounded in terms of $u, \alpha, \beta$. By (10.3) we see that $P$ becomes arbitrarily large by choosing $n$ sufficiently large. Choose $n$ so large that (10.2) is satisfied for $p=P$. Then the assertion follows from Lemma 10.1.

Acknowledgements. The authors are indebted to the referee for his suggestions and remarks which sharpened some of the assertions and improved the exposition of the paper. They thank Shanta Laishram for pointing out an error in an earlier version of Theorem 1.2, and Sierk Rosema, Ionica Smeets and Shanta Laishram for executing some computer calculations for them.

This work was done when the first author was visiting the Department of Mathematics of Leiden University in December 2007-March 2008. He would like to thank the Department for its excellent hospitality and The Netherlands Organisation for Scientific Research (NWO) and the mathematics cluster DIAMANT for their support.

\section{References}

[1] M. Allen and M. Filaseta, A generalization of a second irreducibility theorem of I. Schur, Acta Arith. 109 (2003), 65-79.

[2] —, 一, A generalization of a third irreducibility theorem of I. Schur, ibid. 114 (2004), 183-197.

[3] M. Bauer and M. A. Bennett, Prime factors of consecutive integers, Math. Comp. 77 (2008), 2455-2459.

[4] H. Davenport, Multiplicative Number Theory, Markham, Chicago, 1967.

[5] M. G. Dumas, Sur quelques cas d'irréductibilité des polynomes à coefficients rationnels, J. Math. Pures Appl. 2 (1906), 191-258.

[6] P. Dusart, The $k$ th prime is greater than $k(\ln k+\ln \ln k-1)$ for $k \geq 2$, Math. Comp. 68 (1999), 411-415.

[7] - Inégalités explicites pour $\psi(X), \theta(X), \pi(X)$ et les nombres premiers, $\mathrm{C}$. R. Math. Acad. Sci. Soc. R. Can. 21 (1999), 53-59.

[8] M. Filaseta, The irreducibility of all but finitely many Bessel polynomials, Acta Math. 174 (1995), 383-397. 
[9] M. Filaseta, A generalization of an irreducibility theorem of I. Schur, in: Analytic Number Theory: Proc. Internat. Conf. in honor of H. Halberstam, Vol. 1, B. C. Berndt, H. G. Diamond and A. J. Hildebrand (eds.), Birkhäuser, Boston, 1996, 371-396.

[10] M. Filaseta, C. Finch and J. R. Leidy, T. N. Shorey's influence in the theory of irreducible polynomials, in: Diophantine Equations, N. Saradha (ed.), Narosa, New Delhi, 2008, 77-102.

[11] M. Filaseta, T. Kidd and O. Trifonov, Laguerre polynomials with Galois group $A_{m}$ for each $m$, submitted.

[12] M. Filaseta and T.-Y. Lam, On the irreducibility of the generalized Laguerre polynomials, Acta Arith. 105 (2002), 177-182.

[13] M. Filaseta and O. Trifonov, The irreducibility of Bessel polynomials, J. Reine Angew. Math. 550 (2002), 125-140.

[14] C. E. Finch and N. Saradha, On the irreducibility of certain polynomials with coefficients as products of terms in an arithmetic progression, Acta Arith. 143 (2010), 211-226.

[15] F. Hajir, Some $A_{n}$-extensions obtained from generalized Laguerre polynomials, J. Number Theory 50 (1995), 206-212.

[16] —, Algebraic properties of a family of generalized Laguerre polynomials, Canad. J. Math. 61 (2009), 583-603.

[17] A. E. Ingham, On the difference between consecutive primes, Quart. J. Math. Oxford 8 (1937), 255-266.

[18] S. Laishram, Some topics in number theory (refinements, extensions and generalisations of a theorem of Sylvester on the prime factors of a product of consecutive integers), Ph.D. thesis, TIFR, Mumbai, India, 2007, 152 pp.

[19] S. Laishram and T. N. Shorey, Number of prime divisors in a product of consecutive integers, Acta Arith. 113 (2004), 327-341.

[20] - - - The greatest prime divisor of a product of consecutive integers, ibid. 120 (2005), 299-306.

[21] L. J. Lander and T. R. Parkin, On first appearance of prime differences, Math. Comp. 21 (1967), 483-488.

[22] D. H. Lehmer, On a problem of Störmer, Illinois J. Math. 8 (1964), 57-79.

[23] H. Robbins, A remark on Stirling's formula, Amer. Math. Monthly 62 (1955), 26-29.

[24] J. B. Rosser and L. Schoenfeld, Approximate formulas for some functions of prime numbers, Illinois J. Math. 6 (1962), 64-94.

[25] N. Saradha and T. N. Shorey, Almost squares and factorizations in consecutive integers, Compos. Math. 138 (2003), 113-124.

[26] I. Schur, Einige Sätze über Primzahlen mit Anwendungen auf Irreduzibilitätsfragen I, Sitzungsber. Preuss. Akad. Berlin Phys.-Math. Kl. 14 (1929), 125-136.

[27] —, Einige Sätze über Primzahlen mit Anwendungen auf Irreduzibilitätsfragen II, ibid., 370-391.

[28] —, Affektlose Gleichungen in der Theorie der Laguerreschen und Hermiteschen Polynome, J. Reine Angew. Math. 165 (1931), 52-58.

[29] E. A. Sell, On a certain family of generalized Laguerre polynomials, J. Number Theory 107 (2004), 266-281.

[30] T. N. Shorey, On gaps between numbers with a large prime factor, II, Acta Arith. 25 (1974), 365-373.

[31] T. N. Shorey and R. Tijdeman, On the greatest prime factor of an arithmetical progression (II), ibid. 53 (1990), 499-504. 
[32] T. N. Shorey and R. Tijdeman, Perfect powers in products of terms in an arithmetical progression III, ibid. 61 (1992), 391-398.

T. N. Shorey

School of Mathematics

Tata Institute of Fundamental Research

Homi Bhabha Road

Mumbai 400005, India

E-mail: shorey@math.tifr.res.in
R. Tijdeman Mathematical Institute Leiden University P.O. Box 9512 2300 RA Leiden, The Netherlands E-mail: tijdeman@math.leidenuniv.nl

Received on 1.10.2008

and in revised form on 8.7.2010 